\title{
Mastopexy Autoaugmentation by Using Vertical and Triangular Flaps of Mammary Parenchyma Through a Vertical Ice Cream Cone-Shaped Approach
}

\author{
Nina S. Naidu ${ }^{1}$ (D)
}

Received: 27 February 2019/Accepted: 5 March 2019/Published online: 19 March 2019

(C) Springer Science+Business Media, LLC, part of Springer Nature and International Society of Aesthetic Plastic Surgery 2019

Level of Evidence $V$ This journal requires that authors assign a level of evidence to each article. For a full description of these Evidence-Based Medicine ratings, please refer to the Table of Contents or the online Instructions to Authors www.springer.com/00266.

\section{Keywords Mastopexy · Autoaugmentation · Vertical} mastopexy

In "Mastopexy Autoaugmentation by Using Vertical and Triangular Flaps of Mammary Parenchyma Through a Vertical Ice Cream Cone-Shaped Approach," the authors present a novel flap-based technique for autoaugmentation during mastopexy [1]. They present data on 86 breasts of 43 patients with mild to moderate breast ptosis and hypertrophy, with an extended follow-up period of two years demonstrating excellent maintenance of results.

Both specific markings and operative technique have been presented. Markings are commenced by marking a vertical line from the mid-clavicle to the nipple. The intersection of this line with the transposed infra-mammary fold on the breast creates point $A$. A circle with a diameter measuring $8-10 \mathrm{~cm}$ is then marked with $A$ at the highest point of the circle, and point $\mathrm{D}$ at the most inferior point. The transverse diameter of the circle, line $\mathrm{BC}$, is displaced $1-2 \mathrm{~cm}$ distally to create line $\mathrm{B}^{1} \mathrm{C}^{1}$ and point $\mathrm{D}^{1}$. Note that the diameter of the circle and the amount of displacement will be determined by the degree of breast ptosis and

\footnotetext{
Nina S. Naidu

drnaidu@naiduplasticsurgery.com

1021 Park Avenue, New York, NY 10028, USA
}

hypertrophy, with presumably greater dimensions for larger and more ptotic breasts. The markings for the vertical aspect of the incision are created by displacing point $\mathrm{D}^{1}$ inferiorly to the infra-mammary fold to set a new point of $\mathrm{D}^{2}$. The length from $\mathrm{D}^{1}$ to $\mathrm{D}^{2}$ will measure $6-7 \mathrm{~cm}$. From $\mathrm{D}^{2}$, two curved lines are drawn to points $\mathrm{B}^{1}$ and $\mathrm{C}^{1}$, with the degree of concavity of the curve increasing with greater breast hypertrophy. This then creates the described "vertical ice cream cone-shaped" markings.

The operative technique itself begins with de-epithelialization of the peri-areolar markings, thereby creating a superior pedicle for supply of the nipple-areolar complex. The skin within the vertical markings is resected. Three separate flaps are now created, beginning with a vertical rectangular flap (based on the inferior pedicle) which extends from the infra-mammary fold superiorly along the lower pole and underneath the areola, terminating at its upper border. A subglandular tunnel is created superiorly, and the apex of the flap is secured at the third rib, while the corners of the flap are fixed at the serratus and pectoralis fascia. Two oblique flaps are then created from the vertical pillars, which are then brought down to the lower pole and advanced over each other to create a supportive girdle. These flaps are secured to the pectoralis fascia and each other.

The authors have addressed two critical and persistent issues with conventional mastopexy in their paper. First, they recognize that simply lifting the breast is not enough; there must be an adequate fill of the upper pole. Traditional methods for fill of the upper pole have included implant placement, which creates additional supply and screening costs for the patient, and fat grafting, which requires additional operative time and an adequate donor site for harvest. Autoaugmentation techniques include the use of dermal suspension [2], the pectoralis sling [3], and turnover 
of the superior pedicle [4]. While all methods address the upper pole, they do not always guide us on an oft-overlooked component of mastopexy, that of the reshaping and securing the lower pole. If the lower pole of the breast in mastopexy is used solely as the source of fill without attention to its residual volume or shape, the breast will be left with too much volume in the upper pole relative to the reduced lower pole, creating in effect a "tuberous" shape to the breast. The authors are to be commended for providing a solution to this potential pitfall.

One could argue that this is not a controlled study, which would demand that one breast receive a standard mastopexy while the other breast undergoes the described autoaugmentation. However, those of us who have performed many breast lifts will hopefully have the humility to acknowledge that the authors' results exhibit far better longevity than that to which many of us can claim, with not a single vertical incision extending beyond $8 \mathrm{~cm}$.

Although the authors have described their technique clearly and have illustrated well both the markings and dissection, I suspect that successful execution of their method-like that of all good flap designs-requires a certain learning curve. A video demonstrating both the markings and the operative technique would be most useful for anyone who wishes to incorporate this technique into his or her practice. The curved markings on the lower pole may be similar to those used when displacing the breast laterally and medially, as described by Hall-Findlay [5], and this would benefit from clarification. Although complications were not described, I do question whether or not there is a risk of fat necrosis in the two smaller flaps which are used to buttress and shape the lower pole, as it is not clear if the blood supply from presumably the intercostal perforators would be sufficient. Small flaps, and their tips, tend to be fickle friends.
Finally, the authors' presented postoperative results do not have a rounded upper pole or augmented look, but this is far from criticism; the results show a feminine and natural shape with which most patients would be delighted. I thank the authors for presenting us with a novel addition to the surgical armamentarium for the treatment of breast ptosis.

\section{Compliance with Ethical Standards}

Conflict of interest The author is a shareholder in Ideal Implant, Incorporated.

Human and Animal Rights Statement This article does not contain any studies with human participants or animals performed by any of the authors.

Informed Consent For this type of study informed consent is not required.

\section{References}

1. Abramo AC, Lucena TW, Sgarbi RG et al (2019) Mastopexy autoaugmentation by using vertical and triangular flaps of mammary parenchyma through a vertical ice cream cone-shaped approach. Aesthetic Plast Surg. https://doi.org/10.1007/s00266019-01337-1

2. Rubin JP (2006) Mastopexy after massive weight loss: dermal suspension and total parenchymal reshaping. Aesthetic Surg J $26: 214-222$

3. Graf R, Biggs TM (2002) In search of better shape in mastopexy and reduction mammaplasty. Plast Reconstr Surg 110(1):309-317

4. Casas L (2010) Superior pedicle autoaugmentation mastopexy: a review of 34 consecutive patients. Aesthetic Surg J 30:201-210

5. Hall-Findlay E (2002) Vertical breast reduction with a mediallybased pedicle. Aesthetic Surg J 22:185-194

Publisher's Note Springer Nature remains neutral with regard to jurisdictional claims in published maps and institutional affiliations. 